\title{
Elementary Logical Reasoning in the SOM Output Space
}

\author{
Jorge Ramón Letosa ${ }^{\star \star}$ and Timo Honkela \\ Adaptive Informatics Research Centre \\ Aalto University of Science and Technology \\ P.O. Box 15400, FI-00076 Aalto
}

\begin{abstract}
In this paper, we consider how to represent world knowledge using the self-organizing map (SOM), how to use a simple recurrent network (SRN) to device sentence comprehension, and how to use the SOM output space to represent situations and facilitate grounded logical reasoning.
\end{abstract}

\section{Introduction}

The self-organizing map (SOM) is a well known neural network algorithm. The map is typically a 2-dimensional array, the nodes of which become specifically tuned to various input signal patterns or classes of patterns in an orderly fashion [1. The SOM provides a means for modeling concept formation and symbol grounding 23. It also can be used for implicit representation of conceptual hierarchies [1. In this paper, we focus on the question how to conduct basic logical reasoning within the SOM framework. Moreover, we wish to connect the level of representing knowledge propositionally with its representation in the form of natural language sentences. The framework would also allow multimodal grounding of the knowledge (see e.g. 4]) but we do not address the issue here.

In a related work, Duch has provided an overall view and motivation on neurocognitive modeling of linguistic processing [5]. In relation to conceptual modeling, he states that each node in a neurally plausible semantic network is a neural circuit, with similarities and associations between concepts resulting from sharing some common elements or mutual activations that are responsible for semantic priming [5]. Consistent with this line of thought, Miikkulainen developed already some time ago a system for story comprehension using the SOM as a conceptual memory [6]. Later, the model has been extended to include recurrent processing of sentence structures [7. In a recent work, Mayberry and Miikkulainen have introduced a new connectionist model called InSomNet [8]. Their results show that InSomNet learns to represent semantic dependencies accurately and generalizes to novel structures. The InSomNet system interprets sentences nonmonotonically, generating expectations and revising them, priming

\footnotetext{
* The work has been supported by the Academy of Finland.

** Visiting from the University of Zaragoza, Spain. 
future inputs based on semantics, and properly coactivating multiple interpretations of expressions 8 .

Frank has presented a model in which basic logical operations can be represented as a certain kind of Venn diagram manipulations on the SOM surface [9]. Our current work is closely related to Frank's approach. We verify his experimental results, provide some extensions, and build further the underlying theoretical framework. Starting with a model basically focused in the use of self-organizing maps to carry out knowledge representation based on the comprehension of situations from a story created from the combination of basic propositions and the study about how to extract inferences from this representation [10, Frank adds a sentence comprehension model [119] based on a microlanguage that intends to learn how to relate a set of input sentences with the microworld situations represented onto a SOM map, and accomplish some kind of abstraction learning in the sentence comprehension.

As the main objectives in this paper, we consider how to represent propositionally defined situations using the SOM, how to represent the processing and structural representation of related sentences using recurrent neural networks, and how to evaluate the comprehensibility of the sentences in the framework of this methodology. We also consider how logical reasoning can be conducted using the SOM output space as grounding for the propositional elements. We present the underlying theory and methodology as well as an illuminative experiment.

\section{Methods}

The objective of this work is threefold: (1) to represent world knowledge using the self-organizing map (SOM), (2) to use a simple recurrent network (SRN) to device sentence comprehension, and (3) to use the output space to represent situations and facilitate grounded logical reasoning. We will apply set theory and fuzzy set theory [12, clause logic, neural networks (specifically simple recurrent neural networks [13] and the self-organizing map [1]).

The degree of fit between an input value and a particular prototype in the SOM is interpreted as an membership value in the fuzzy set theory. We also assume that the proportional size of the distribution on the map related to a specific input approximates the probability of this input. Furthermore, we assume that the distributions on the map can be interpreted as fuzzy Venn diagrams allowing for basic set-theoretic operations (see e.g. 14]). Venn diagrams show possible logical relations among a finite collection of sets.

Simple recurrent network is a variant multilayer perceptrons. In a simple recurrent network, a set of context units is added to the three-layer network. The connections from the hidden layer to context units allow the network to model time-dependent phenomena and perform tasks like sequence prediction. 13.

\subsection{World Knowledge Representation}

In order to implement world knowledge, we shall begin by constructing a defined microworld framework in which a set of situations takes place. After defining the 
Table 1. Examples of the basic events in the microworld

\begin{tabular}{cll}
\hline No. & Name & Meaning \\
\hline 1 & $70 \mathrm{~s}$ & In the 70s decade. \\
2 & $80 \mathrm{~s}$ & In the 80s decade. \\
3 & lcSuccess & LC gets success. \\
.. & $\ldots$ & $\ldots$ \\
15 & bdHome & BD is at the home country. \\
16 & bdEurope & BD is in Europe. \\
17 & accompanied & He is/they are accompanied. \\
\hline
\end{tabular}

microworld and its constraints, we explain how the microworld knowledge can be represented by means of the help of the Self-Organizing Maps.

Following the model presented in [10], the knowledge of a microworld is learned implicitly by means of training a set of example situations that fulfill the microworld definition; each one of them is created from combining the events such as presented in Table 1. Thus, the SOM output of the model is expected to reflect the inherent regularities, constraints and the a priori probabilities of occurrences of the concrete events within the microworld. This serves as a kind of "experience" the system has after being trained with a set of microworld example situations.

After training, a situation in the microworld is represented by a high-dimensional vector of $n=150$ components (a SOM map). This implementation in which a situation is contained in a vector of fixed size of $n$ elements allows a representation in which $n$ is independent of the size of the example set, although, at the same time, by reducing the dimensionality from $k$, size of the example set, 227 in this case, to $n=150$, some of the information contained in the previous example situations set may be lost. A situation vector is in the form $s(p)=\left(s_{1}(p), s_{2}(p), \ldots, s_{n}(p)\right)$, where every $s_{i}(p)$ has a value between 0 and 1 for every event $p$ ( $p$ can, in fact, be any combination of events) that indicates the extent to which the component, or more precisely, the cell in the SOM, is a part of the representation of $p([9])$. As it can be observed in Fig. 1, the pattern representing "lcHome" overlaps with the pattern in which "lcWrites", showing that if LC is writing a book, LC is at the home country.

In general, a situation can be represented on a map so that it is possible to obtain the probability of occurrence of such situation and any combination of

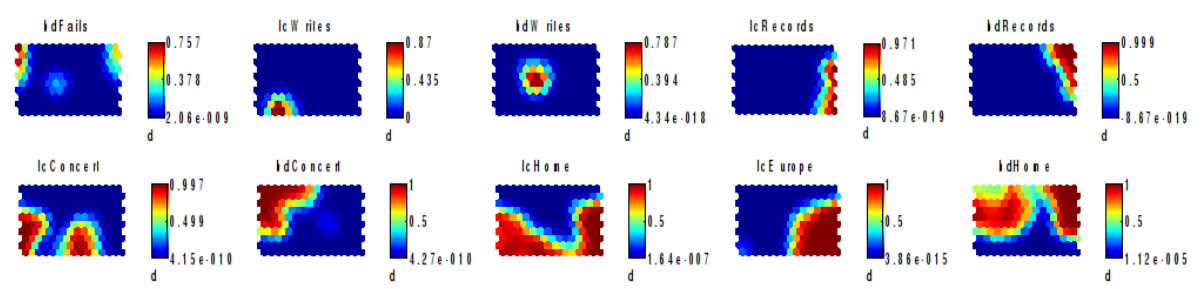

Fig. 1. A subset of the component planes of a situation map 


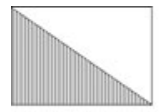

$p$

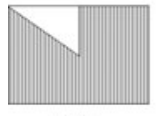

pose

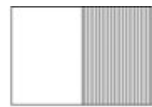

व

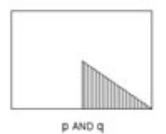

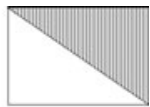

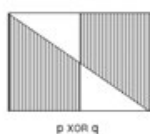

Fig. 2. Basic logical reasoning at the SOM output level

situations by means of fuzzy set theory. Taking the example shown in Fig. 2 where $p$ and $q$ are situations with a probability of 0.5 each one in the microworld and taking into account the equations $s_{i}(\neg p)=1-s_{i}(p)$ and $s_{i}(p \wedge q)=s_{i}(p) s_{i}(q)$ every logical combination of situations can be represented.

Since there is no one-to-one correspondence between propositions and dimensions in the taken representation of situations, we use belief values in order to figure out the results that are given as situation vectors. Let $X=\left(x_{1}, x_{2}, \ldots, x_{n}\right)$ be a situation vector, with $n$ as the number of situation-space dimensions. As a result of training the SOM, the probability (the probability estimate is approximated) that situation $X$ occurs in the microworld equals the fraction of the map it covers (9]). The belief values $\tau$ for a situation $X$ and for a situation $p$ in $X$ are defined as:

$$
\tau(X)=\frac{1}{n} \Sigma_{i} x_{i} . \quad \tau(p \mid X)=\frac{\Sigma_{i} s_{i}(p) x_{i}}{\Sigma_{i} x_{i}}
$$

\subsection{Sentence Comprehension}

Next, we convert sentences from a microlanguage to the representation of the corresponding microworld situation.

The microlanguage used in the experiments consists of 13 words: $L C, B D$, and, is, gets_success, fails, performs_concert, writes_book, records_CD, at_home, in_Europe, single, accompanied. With these words and following the rules of the grammar in Table 2, 396 different sentences can be obtained by associating each of them with a microworld situation.

Microlanguage sentences are transformed into situational vector representations by training a simple recurrent neural network [13. The input layer of the network consists of 13 units, one for each word in the microlanguage. The hidden layer has 50 units and the output layer 150 units, one for each dimension of the situational space.

The words of a sentence are processed one-by-one, so only one of the input units is active at any moment. This way, the output of the network can be observed at any moment and it is possible to analyze how the representation of the situations gets defined as the sentence gets completed.

Belief values are useful in measuring the performance of the model after the network has been trained. The belief value of a situation $X(p)$, represented by 
Table 2. Grammar of the microlanguage

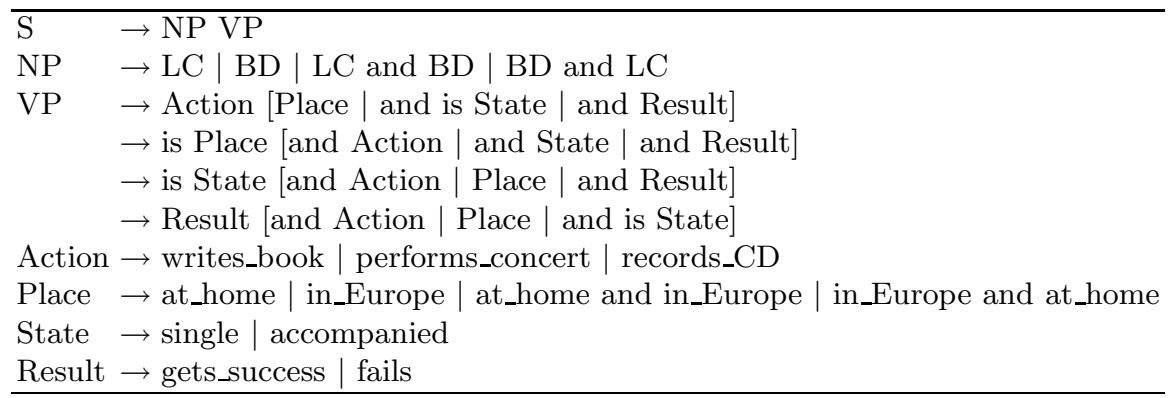

the output for a certain sentence, should be larger than the a priori belief value of the situation $p$ corresponding to that sentence:

$$
\operatorname{compr}(p)=\frac{\tau(p \mid X(p))-\tau(p)}{\tau(p \mid p)-\tau(p)} .
$$

When $\tau(p \mid X(p))>\tau(p)$, the comprehension score is higher than 0 and the network reflects a comprehension of the sentence, having the ideal case when $\tau(p \mid X(p))=\tau(p \mid p)$, and the score is 1 . On the other hand, when the score is negative the network is misunderstanding the sentence and there is no comprehension when the result equals 0 .

\section{$3 \quad$ Experiments}

In the following, we describe the experiments conducted in this work including both the formation of the self-organizing map of the situations and simple recurrent network model of the sentences describing the situations in a microworld.

The example situations set constitutes the input of the SOM and consists of 227 example situations that follow the microworld constraints so that every situation in this set is obtained by one or more propositions combined. Each of the input example situations is a vector $\mu=\left(\mu_{1}, \mu_{2}, \ldots, \mu_{m}\right), m=17$, which is the number of events in the microworld, so that a component $\mu_{i}$ is equal to 1 if it is the case of the example situation or 0 if it is not.

The SRN network is trained with a set of 368 sentences that are presented to the network randomly. The rest of the sentences are not trained and they are used as a test set. After training and calculating the comprehension scores for the sentences present in the training set, the percentage of comprehended sentences is $89.7 \%$ and the mean of the score is 0.3931 . In the other hand, a set of 20 sentences not presented to the network during the training but referring to situations in the microworld that were already present in the training set are considered as well, obtaining a score mean of 0.3330 within the 18 of the 20 sentences that were comprehended. Finally, a set of 8 sentences not presented 
describing new situations resulted in a score mean of 0.3799 and 7 of this set of 8 sentences had a comprehension score above 0. Furthermore, the results show how the short sentences lead to higher comprehension scores than the long ones.

\section{Conclusions}

In this paper, we have described an approach which enables representation of situations in a microworld using the self-organizing map algorithm and processing of sentences that describe the situations using simple recurrent network. We have also considered how to conduct basic logical reasoning at the output level of the self-organizing map in a Venn diagram like manner. The approach seems to facilitate well a grounded approach for modeling story comprehension. The present study is based on a microworld in which the number of different situations and sentences is small. Future research is needed to test how well the methodology scales up to large real-world applications.

\section{References}

1. Kohonen, T.: Self-Organizing Maps. Springer, Heidelberg (2001)

2. Gärdenfors, P.: Conceptual spaces: The Geometry of Thought. MIT Press, Cambridge (2000)

3. Honkela, T.: Self-Organizing Maps in Symbol Processing. In: Hybrid Neural Systems, pp. 348-362. Springer, Heidelberg (2000)

4. Harnad, S.: The symbol grounding problem. Phys. D 42(1-3), 335-346 (1990)

5. Duch, W.: Neurocognitive informatics manifesto. In: Proc. of IMS 2009, pp. 264282 (2009)

6. Miikkulainen, R.: Subsymbolic Natural Language Processing: An Integrated Model of Scripts, Lexicon, and Memory. MIT Press, Cambridge (1993)

7. Mayberry, M.R., Miikkulainen, R.: SARDSRN: a neural network shift-reduce parser. In: Proc. of IJCAI 1999, pp. 820-825. Morgan Kaufmann, San Francisco (1999)

8. Mayberry, M.R., Miikkulainen, R.: Incremental nonmonotonic sentence interpretation through semantic self-organization. Technical Report AI08-12, Department of Computer Sciences, University of Texas at Austin

9. Frank, S.L.: Sentence comprehension as the construction of a situational representation: A connectionist model. In: Proceedings of AMKLC 2005, International Symposium on Adaptive Models of Knowledge, Language and Cognition, pp. 27 33. Helsinki University of Technology, Espoo (2005)

10. Frank, S.L., Koppen, M., Noordman, L.G.M., Vonk, W.: Modeling knowledgebased inferences in story comprehension. Cognitive Science 27(6), 875-910 (2003)

11. Frank, S.L.: Sentence comprehension without propositional structure. In: Modeling language, cognition and action, pp. 119-128. World Scientific, New Jersey (2005)

12. Zadeh, L.A.: Fuzzy sets. Information and Control 8, 338-353 (1965)

13. Elman, J.L.: Finding structure in time. Cognitive Science 14(2), 179-211 (1990)

14. Harris, J.: Fuzzy Logic Applications in Engineering Science. Springer, Dordrecht (2006) 